\title{
HISTORY \\ Contributions of the University of Cape Town to medical science in the first 100 years: Personal reflections
}

\author{
Stuart Saunders
}

The contributions of the University of Cape Town's Faculty of Health Sciences to medical science through research over the past 100 years are reviewed. The application of contemporary techniques to common medical problems in the developing world had important implications globally. The Faculty can be proud of its achievements in many areas important to the health of people in South Africa, Africa and beyond.

S Afr Med J 2012;102(6):391-393.
The development of the Xenopus laevis test for pregnancy was perhaps the most outstanding early research in the Medical School.

\section{Joint agreement}

It was the advent of the Joint Agreement with the Cape Provincial Administration in 1950 that resulted in posts for full-time specialists in the Faculty; that gave a real boost to research. This was against the background of the establishment of the Medical and Dental Research Committee of the Council for Scientific and Industrial Research (CSIR) and later of the Medical Research Council (MRC) which significantly helped to provide the finances for research. Brock, regarded by many as the father of medical research in South Africa, put it this way: 'The most significant effect of [the joint agreement] has been that [people] of high academic achievement and research potential are more frequently being attracted into full-time appointments. There they can devote their thinking to the raising of standards and the advance of knowledge without the inevitable distractions of private practice.'

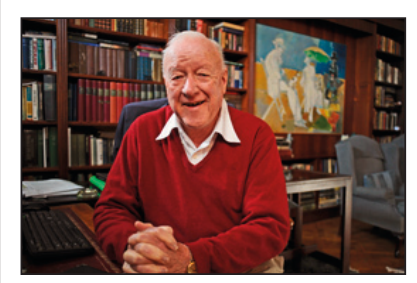

Emeritus Professor Stuart J Saunders (GCOB, MB ChB (Hons), MD, FCP (SA), FRCP (Lond), FRSSAf) qualified in medicine at the University of Cape Town (UCT) in 1953. He did postgraduate work at the Royal Post-Graduate Medical School at Hammersmith in London and at Harvard University in Boston. He became a specialist physician in the UCT Department of Medicine in 1960; he served as the Head of the Department from 1971 to 1980, and was appointed as Vice-Chancellor until his retirement in 1996. He enjoyed a distinguished academic career, publishing 190 scientific articles. He founded the Liver Research Group (now Liver Research Centre) at UCT. He is the recipient of Honorary Doctorates from the Universities of Aberdeen, Toronto, Sheffield, Princeton and Cape Town, and of Rhodes University, and is an Honorary Fellow of the Colleges of Medicine (SA). In his retirement, he currently serves as Trustee of the Leon Foundation, Fox Foundation, Webb Trust and UCT Trust (UK), and is senior adviser to the A W Mellon Foundation of New York. Until recently, he continued to practise as a consultant physician and specialist in liver diseases at Groote Schuur Hospital. His memoirs, Vice-Chancellor on a Tightrope, were published by David Phillip in 2000. He received the award of Grand Counsellor of the Order of the Baobab from the President of South Africa in 2002.

\section{Broad research on common diseases}

One of the reasons for the international recognition that research at the Medical School enjoyed was that it exploited the conjunction of the use of sophisticated techniques with a constellation of diseases infrequently seen in the developed world, but with results which were of universal importance.

Over the next 30 years this resulted in:

- Medicine: important contributions in cardiology in the diagnosis and treatment of rheumatic heart disease, tuberculous pericarditis, congenital heart disease and arrythmias; in respiratory medicine, research in asthma and chronic obstructive airways disease; in endocrinology, the characterisation and detection of pre-diabetes, the measurement of exophthalmos promoting factor, and studies of pituitary and hypothalamic function; in nutrition, stimulated by the creation of the Clinical Nutrition Unit, the impact of diet on the plasma cholesterol and the initiation of an appreciation of the role of serum cholesterol in ischaemic heart disease, albumin synthesis and albumin metabolism using sophisticated isotope techniques, a study of the anaemia of scurvy, and the pathogenesis of and metabolic abnormalities in protein calorie malnutrition; in gastroenterology, the study of gastric and pancreatic function with large-scale studies of the diagnosis and treatment of peptic ulceration and acute and chronic pancreatitis; in liver disease, controlling factors in liver regeneration and the treatment of acute liver failure with exchange transfusion, isolated liver perfusion and crosscirculation; clinical and biochemical studies in porphyria, establishing new methods of diagnosis and treatment; and in haematology, bone marrow transplantation and its immunological consequences, coagulation defects with new insights into the coagulation cascade, and the diagnosis and treatment of multiple myeloma.

- Surgery: intrauterine vascular interference to the small bowels of dogs in studying the pathogenesis and treatment of small bowel atresia and the initiation of intrauterine management of certain congenital anomalies; study of anatomy and successful separation of conjoined twins; developments in burn management; peripheral vascular disease and one of the earliest developments of plethysmography; studies in portal hypertension and sclerotherapy; bile duct carcinoma and liver transplantation in animals and humans; the elucidation of the cause of malignant hyperpyrexia and its successful treatment; and, of course, the climactic heart transplantation and associated work in immunology. 
- Paediatrics: intellectual and growth stunting in malnutrition; studies in asthma; and the treatment of tetanus with ventilation.

- In the broad field of biochemistry: work on calcium pumps in skeletal muscle, GnRH and its receptors across all animal families, LDL receptors on mini-albumins and cytochrome $\mathrm{P} 450$; and in virology, poliomyelitis, blue tongue disease and bacteriophages.

- Pathology: the nephrotic syndrome and cardiac rejection.

- Human genetics: the study of skeletal abnormalities.

\section{Post heart transplant}

The success of the heart transplant resulted in the erection and equipping of the Heart Disease and Organ Transplantation building - aimed at institutionalising this achievement and boosting research. This resulted in work in clinical immunology and access to a modern animal house. The new facilities, plus the injection of funds by the Chris Barnard fund, greatly assisted the development of studies, among others, of the adverse effects of glucose deprivation on the ischaemic heart. Furthermore, it allowed the demonstration that exercise-trained rats had increased resistance to sudden death by ventricular fibrillation, and furthered research into heart muscle metabolism. This ultimately resulted in the establishment of the Hatter Institute for the study of heart disease. Another result was the formation of a research group into fundamental aspects of exercise, leading also to important work in muscle metabolism.

This burst of research in the 1950s - 1970s was also due to the relationship between the Medical School and the province; the latter understanding that good research resulted in first-class clinical care and standards. Funds were reasonably available and the border between routine clinical care and research was blurred. The role of what Opie has termed the 'PhysicianScientist' (L H Opie, personal communication), using physician in the broad sense, was critical. To quote Opie further: 'Overall I think the major point was [that] the atmosphere in GSH was electrifying (in 1970s), one felt that new work was being done in every direction and that we were all proud of being on the GSH staff. Into this mix must be added the fact that there was real collegiality in the medical school and teaching hospitals with positive interaction and mutual support which was important.' The atmosphere and the culture of institutions bears a direct influence on research productivity.

\section{Lesser provincial role}

In the 1980s, 1990s and early part of the 21 st century, the Faculty built on the tradition that had been established, despite the challenges presented by a much less favourable financial environment with shrinking provincial budgets for teaching hospitals, and a loss of appreciation - on the part of the province - of the importance of research in patient care. Posts were frozen and wards mothballed. On top of this must be added the profound effects of the HIV/AIDS epidemic. It is greatly to the credit of staff that research productivity was maintained.

Among the highlights of this period was the elucidation of the molecular genetics of variegate porphyria and other aspects of research in porphyria; the description of the mechanism of myocardial protection by insulin and other experimental studies in the treatment of and protection from myocardial infarction; the definition and clinical characterisation of tuberculosis (TB)associated immune reconstitution disease; kidney transplantation between HIV-positive donors and recipients; the improvement of community diagnosis and care of patients with lung disease and HIV; research in allergology and drug-resistant TB; studies in all aspects of HIV and AIDS including the development of a vaccine; bioethics and the emphasis on ethical standards; a national centre of drug safety, the development of traditional medicines, a role in the global eradication of poliomyelitis and the development of artesunate for life-threatening malaria in infants and children; vitamin A studies and TB vaccine trials; the genetics of cardiomyopathy at a molecular level; studies in rheumatic fever, tuberculous pericarditis, cervical carcinoma and papilloma virus; further studies on the genetics of colon carcinoma; studies on the revascularisation of synthetic grafts; and the brain and behaviour initiative which, like drug discovery in the Department of Chemistry, is a signature theme at the University of Cape Town (UCT).

\section{New research units and groups}

Much other important research was undertaken, often as part of the programmes of research groups, units and centres supported by the MRC, the Hatter Institute, the Lung Institute and the Desmond Tutu HIV Research Centre.

An important development was the establishment of the Institute of Infectious Disease and Molecular Medicine (IIDMM). This was a further impetus to research. The institute concentrates its research efforts on infectious diseases, particularly HIV/AIDS, TB and noncommunicable diseases prevalent in Africa. There are 20 research groups including the following: the South African (SA) TB Vaccine Initiative, the Wellcome Trust-funded Clinical Infectious Diseases Research Initiative, the Desmond Tutu HIV Centre, 6 research chairs awarded by the SA research chairs initiative, 4 MRC units, 2 MRC research groups and the UCT node of the Department of Science and Technology (DST)/National Research Foundation (NRF) Centre for Excellence in Biomedical Research. Many areas of modern molecular-based research are emphasised including molecular and cell biology, immunology, virology, microbiology, genetic and genomics, biochemistry, pharmacology, vaccinology, molecular epidemiology, and structural, high-throughput computational biology. The IIDMM has 22 full, 18 associate, and 7 adjunct members, over 140 research officers, technical and administrative support staff, 147 masters and PhD students, and 47 post-doctoral fellows.

Major funders of the IIDMM are the Wellcome Trust, the National Institutes of Health (NIH), the Aeras Global TB Vaccine Foundation, the Bill and Melinda Gates Foundation, the European and Developing Countries Clinical Trials Partnership, the NRF and the MRC.

Among the important research achievements at the IIDMM have been: 2 HIV vaccines, currently being tested in South Africa and the USA; the first efficacy trial of a new TB vaccine in infants in 80 years; the first international randomised controlled trial of the HPV bivalent vaccine in HIV-positive women; the use of prednisone in the treatment of the TB-IRIS syndrome; studies on the causal relationship between vitamin $\mathrm{D}$ status and $\mathrm{TB}$ incidence in Cape Town; the evaluation of the GeneXpert Mycobacterium tuberculosis (MTB)/rifampicin (RIF) diagnostic, to screen for HIV-associated $\mathrm{TB}$ and rifampicin resistance before the start of antiviral treatment; studies of the angiotensin-1-converting enzyme; the crystal structure of a type-111 glutamine synthetase; and work on CD4 T-cell depletion at the cervix during HIV infection, showing an association with the accumulation of terminally differentiated T-cells.

\section{Nobel Prize winners}

Special mention must be made of the 2 Nobel Prize winners whose work contributed so much to medicine but who were members 
of the Faculty of Science: Klug (molecular biology) and Cormack (computed tomography (CT) scan).

\section{Private practice}

The jury is out as to whether the advent of private practice for 'fulltime' clinicians has adversely affected their contributions to research. Brock spoke of the 'dead hand of Harley Street' and clearly saw the dangers. There is a difference of opinion among a number of senior members of the clinical departments, with a majority feeling that the influence of private practice has been negative. In this regard, Brock emphasised that the obligations of full-time members of staff were firstly to their patients, secondly to their students, thirdly to research and finally to administration. He believed, and I think correctly so, that all full-time members of staff had an obligation to meet all those goals. The distractions of private practice threaten the fulfilment of these duties. Creative energy, such as that needed in research, is timeconsuming and most likely to suffer first from private practice. Apart from the fact that full-time members of a Faculty of Health Sciences have an obligation to add to knowledge through research - and this does not include pharmaceutical company-driven controlled trials which are often undertaken for material gain - many believe, and I am one of them, that good researchers are often the most stimulating teachers and, in my experience, are usually the best clinicians. There is a clear obligation for deans and heads of departments, now and in the future, to be vigilant of the potentially adverse effects of private practice undertaken by 'full-time' staff, and to ensure that the proud record of research is maintained,

The other threat to the maintenance of high research standards is complacency. Research requires consistent hard work, is often emotionally taxing and is a very challenging enterprise.

The UCT Faculty of Health Sciences has maintained high standards and made important contributions to knowledge over many years. The staff should be congratulated on their achievements.

\footnotetext{
1. Brock JF. The evolution of medical research in South Africa. S Afr Med J 1960;34:420-421.
}

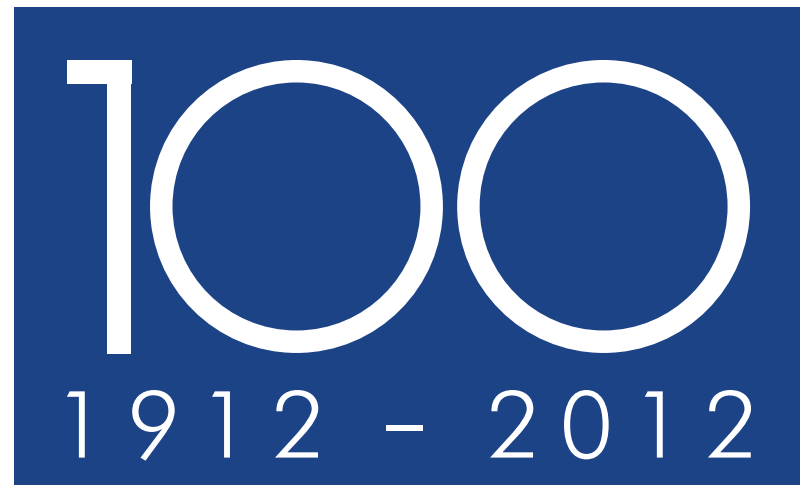

\section{REFLECTING ON THE PAST}

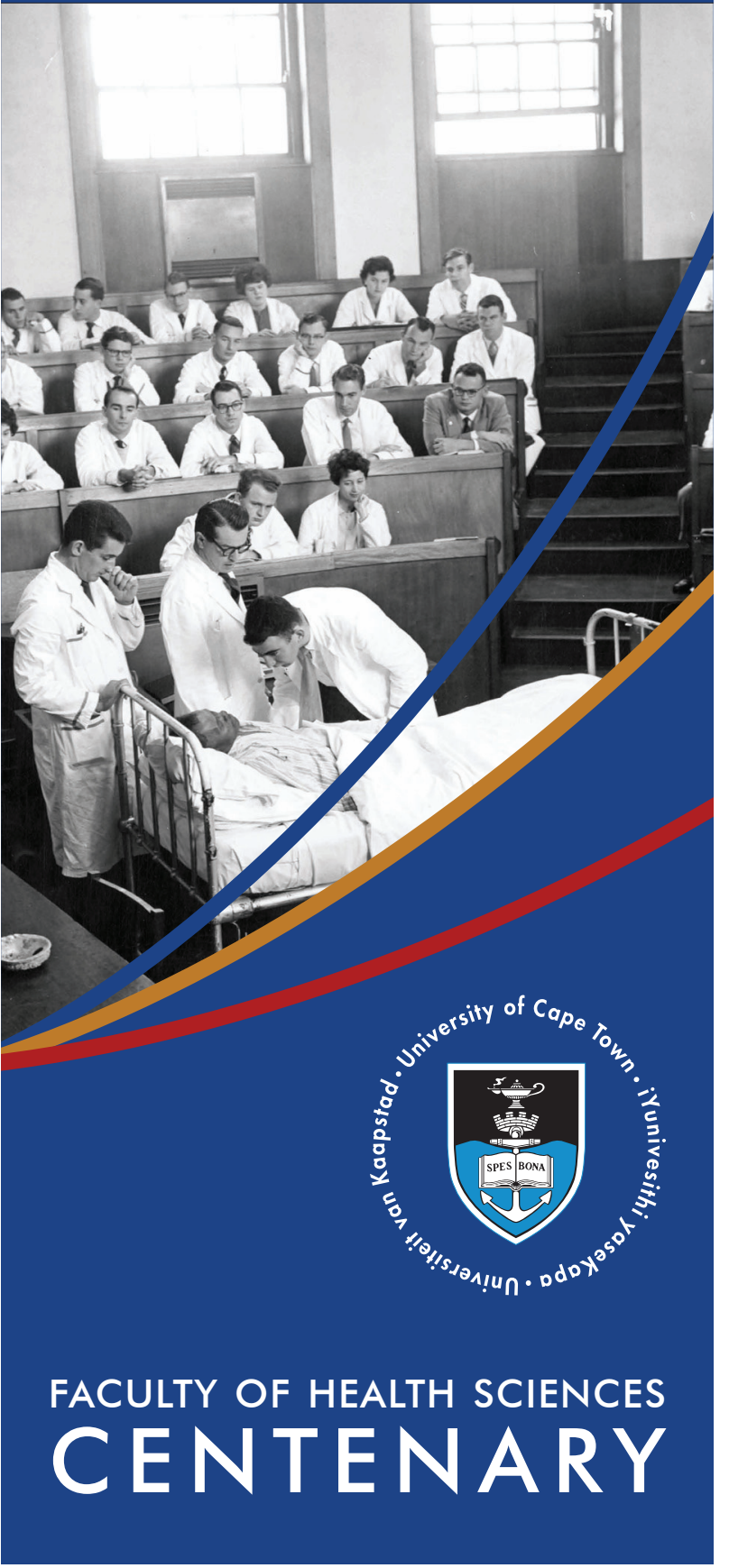

